\title{
DO PLATELET INDICES PLAY A ROLE WITH RESPECT TO PLATELET COUNT AND INFECTIONS?
}

\author{
Rachana Kiran Koppalkar1, Purnima S. Rao², Sandhya I3, Prithal G4
}

1 Postgraduate Student, Department of Pathology, A. J. Institute of Medical Sciences and Research Centre, Mangalore, Karnataka, India. ${ }^{2}$ Associate Professor, Department of Pathology, A. J. Institute of Medical Sciences and Research Centre, Mangalore, Karnataka, India. ${ }_{3}^{3}$ Associate Professor, Department of Pathology, A. J. Institute of Medical Sciences and Research Centre, Mangalore, Karnataka, India. ${ }^{4}$ Assistant Professor, Department of Pathology, A. J. Institute of Medical Sciences and Research Centre, Mangalore, Karnataka, India. ABSTRACT

\section{BACKGROUND}

Platelet an essential constituent of blood plays an important role in coagulation, thrombosis, inflammation and maintains the integrity of vascular endothelial cells. Platelet indices like Plateletcrit (PCT), Mean Platelet Volume (MPV), Platelet Distribution Width (PDW) and Platelet Large Cell Ratio (P-LCR) are a group of derived platelet parameters obtained as a part of automatic complete blood count. Various infections and metabolic disorders cause variations in the platelet counts and platelet indices.

This study aims to summarise the potential role of platelets and platelet indices as risk markers for early screening of infections.

\section{MATERIALS AND METHODS}

This is a descriptive comparative study. The present study included 103 cases and they were subtyped into four groups, i.e. tuberculosis, dengue, malaria and chronic liver disease. Platelet indices in different infections were obtained.

\section{RESULTS}

PDW was normal in dengue, malaria and chronic liver disease, but decreased in tuberculosis. MPV was normal in malaria, dengue and chronic liver disease, but MPV was low in tuberculosis. P-LCR was increased in dengue and malaria, decreased in tuberculosis and normal in chronic liver disease.

\section{CONCLUSION}

Platelet indices have been valuable indicators of illness severity and effective predictors of clinical outcomes. Platelet indices could be easily measured from routine blood examination that is inexpensive and repeatable. This provides a convenient stratification tool in infections.

\section{KEY WORDS}

Infections, Platelet Indices.

HOW TO CITE THIS ARTICLE: Koppalkar RK, Rao PS, Sandhya I, et al. Do platelet indices play a role with respect to platelet count and infections? J. Evolution Med. Dent. Sci. 2018;7(44):4754-4757, DOI: 10.14260/jemds/2018/1061

\section{BACKGROUND \\ Platelets are dynamic blood particles which play an important role in coagulation, thrombosis and also in maintaining the integrity of vascular endothelial cells. These when stimulated undergoes variation in shape and surface area. Platelet indices are biomarkers of platelet activation. The commonly used platelet indices are Mean Platelet Volume (MPV), Platelet Distribution Width (PDW), Plateletcrit (PCT) and Platelet Large Cell Ratio (P-LCR). These indices could be easily obtained by automated analyser. Platelet indices tend to be altered in various infections. This study aims to evaluate these indices in infections, which can lead to early diagnosis and reduce the severity of infection. For example, in malarial infection, platelet count and plateletcrit are effective markers. MPV and PDW showed sensitivity for dengue fever.[1]}

'Financial or Other Competing Interest': None.

Submission 16-09-2018, Peer Review 10-10-2018,

Acceptance 16-10-2018, Published 29-10-2018.

Corresponding Author:

PurnimaS. Rao,

Aadheeshwari Apartments,

Mahamaya Temple Road,

Field Street, Mangalore-575001,

Karnataka, India.

E-mail: pursrao@gmail.com

DOI: $10.14260 /$ jemds $/ 2018 / 1061$
Objective- This study aims to determine the potential role of platelets and platelet indices as risk markers for early screening of infections.

\section{MATERIALS AND METHODS}

This descriptive comparative study was undertaken in a tertiary care centre in coastal Karnataka. A total of 103 cases were studied. Study subjects included 74 thrombocytopenia and 29 thrombocytosis cases. In all the cases age, gender and relevant clinical history were obtained. The thrombocytopenia patients were asked for complaints of fever with chills, cough, headache, bleeding tendencies and organomegaly. Based on clinical diagnosis and relevant laboratory parameters they were subtyped into four groups i.e. tuberculosis, dengue, malaria and chronic liver disease.

In the present study, cases are those with thrombocytopenia and thrombocytosis. Controls are healthy blood donors or patients with normal platelet count.

\section{Inclusion Criteria}

1. Abnormal platelet counts (Thrombocytopenia and Thrombocytosis).

2. Age 18 - 75 years.

\section{Exclusion Criteria}

1. Pregnant women.

2. Patients with active haemorrhage.

3. Patients on antiplatelet drugs or had a blood transfusion. 


\section{Haematological Analysis}

All EDTA-anticoagulated blood samples were obtained to perform complete haemogram analysis using Automated Haematology analyser (Sysmex XN 1000). This analyser provided results of platelet count, MPV, PDW, P-LCR and PCT. The device performed haematology analysis according to electronic impedance method.

The platelet indices were studied in patients with abnormal platelet counts and in various infections. The results obtained were compared with a control group of 102 healthy blood donors with normal platelet parameters.

The normal ranges of MPV, PDW and PCT for this analyser were as follows: Platelet count: $150.00-450.00 \times$ 103/ $\mu \mathrm{L}$; MPV: 8 - 12.4 FL; PDW: 9 - 14\%, PCT: $0.16-0.38 \%$, P-LCR: 15 - 35\% respectively.

\section{Statistical Analysis}

Platelet indices in different infections were expressed as median and Interquartile Range (IQR). A p-value was calculated by Fisher's exact test. Data was analysed by Kruskal-Wallis test. Further analysis was carried out by posthoc Mann-Whitney test, as data was not correlating with normality. A p-value of $<0.05$ was considered statistically significant.

\section{RESULTS}

A total of 103 cases were studied and compared with a control group of 102 individuals. Of the 103 cases, 74 cases (71.8\%) had thrombocytopenia and 29 (28.1\%) had thrombocytosis respectively.

Thrombocytopenia were common among men being $71.6 \%$. Thrombocytosis were seen in both males $(48.3 \%)$ and females (51.7\%).

Platelet parameters like PDW, MPV, P-LCR and PCT were assessed using automated haematology analyser (Sysmex XN 1000). These parameters were compared across three groups, i.e. thrombocytosis, thrombocytopenia and control group.

PDW was low (27.6\%) or normal (69\%) in thrombocytosis patients. PDW was normal (50\%) or high $(27 \%)$ in thrombocytopenia cases. MPV was normal in both thrombocytopenia and thrombocytosis patients. P-LCR was normal in thrombocytopenia and normal/ high in thrombocytopenia patients.

Based on the patient's clinical history they were subdivided into various categories like dengue (5), malaria (14), tuberculosis (3) and chronic liver disease (10) as given in Table/ Figure 1.

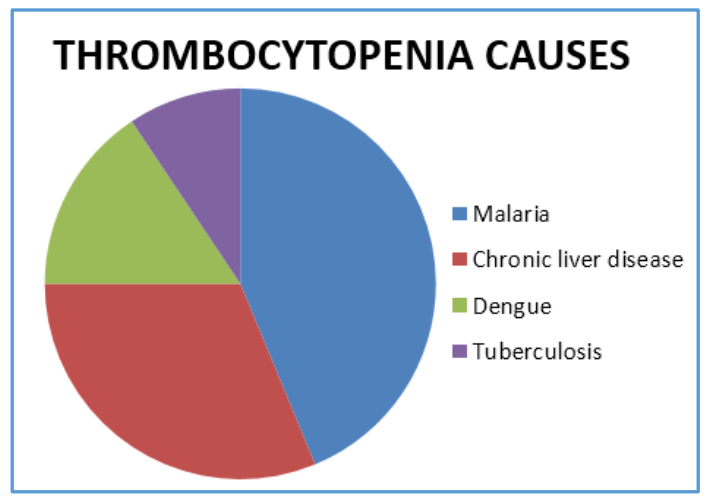

Figure 1. Causes of Thrombocytopenia
The platelet indices (PDW, MPV, P-LCR), median and Interquartile Range (IQR) in different categories are given in Table/ Figure 2, 3, 4.

\begin{tabular}{|c|c|c|c|c|c|}
\hline & \multirow[t]{2}{*}{ History } & \multirow[t]{2}{*}{ Median } & \multicolumn{2}{|c|}{ IQR } & \multirow{2}{*}{\begin{tabular}{|c} 
Kruskal- \\
Wallis Test \\
P-value
\end{tabular}} \\
\hline \multirow[b]{5}{*}{ PDW } & & & 25th & 75th & \\
\hline & Dengue & 13.300 & 12.950 & 22.400 & \multirow{4}{*}{$<0.001$} \\
\hline & Tuberculosis & .00 & .00 & 9.60 & \\
\hline & Malaria & 13.650 & 12.600 & 16.275 & \\
\hline & $\begin{array}{c}\text { Chronic Liver } \\
\text { Disease }\end{array}$ & 12.850 & 11.250 & 17.400 & \\
\hline \multicolumn{6}{|c|}{ Table 2. PDW among different Categories } \\
\hline
\end{tabular}

\begin{tabular}{|c|c|c|c|c|c|}
\hline \multirow{7}{*}{ History } & Median & \multicolumn{2}{|c|}{ IQR } & $\begin{array}{c}\text { Kruskal- } \\
\text { Wallis } \\
\text { Test P- } \\
\text { value }\end{array}$ \\
\cline { 2 - 5 } & & & 25th & 75th & \\
\cline { 2 - 5 } MPV & Dengue & 11.8 & 10.850 & 13.7 & \multirow{4}{*}{$<0.001$} \\
\cline { 2 - 5 } & Tuberculosis & .00 & .00 & 10.0 & \\
\cline { 2 - 5 } & Malaria & 11.7 & 10.600 & 11.925 & \\
\cline { 2 - 5 } & $\begin{array}{c}\text { Chronic Liver } \\
\text { Disease }\end{array}$ & 11.100 & 10.675 & 12.150 & \\
\hline \multicolumn{3}{|c|}{ Table 3. MPV across various Diseases } \\
\hline
\end{tabular}

\begin{tabular}{|c|c|c|c|c|c|}
\hline \multirow{4}{*}{ History } & Median & \multicolumn{2}{|c|}{ IQR } & $\begin{array}{c}\text { Kruskal- } \\
\text { Wallis } \\
\text { Test P- } \\
\text { value }\end{array}$ \\
\cline { 2 - 5 } & & & 25th & 75th & \\
\cline { 2 - 5 } & Dengue & 40.100 & 31.750 & 53.85 & \\
\cline { 2 - 5 } & P-LCR & .00 & 23.5 & \multirow{2}{*}{0.001} \\
\cline { 2 - 5 } & Tuberculosis & .00 & 38.100 & 30.375 & 40.675 \\
\cline { 2 - 5 } & $\begin{array}{c}\text { Chronic Liver } \\
\text { Disease }\end{array}$ & 33.500 & 30.175 & 42.450 & \\
\hline \multicolumn{4}{|c|}{ Table 4. P-LCR in different Diseases } \\
\hline
\end{tabular}

Comparison of platelets indices in different infections in thrombocytopenia patients is given in Table/ Figure 5.

PDW was normal in dengue, malaria and chronic liver disease, but decreased in tuberculosis. MPV was normal in malaria, dengue and chronic liver disease, but MPV was low in tuberculosis. P-LCR was increased in dengue and malaria, decreased in tuberculosis and normal in chronic liver disease.

\begin{tabular}{|c|c|c|c|c|}
\hline \multirow{4}{*}{ PDW } & \multicolumn{2}{|c|}{ Infection Comparison } & \multirow{2}{*}{$\begin{array}{c}\begin{array}{c}\mathbf{P} \\
\text { value }\end{array} \\
0.004\end{array}$} & \multirow{2}{*}{$\begin{array}{c}\text { Significance } \\
\text { HS } \\
\end{array}$} \\
\hline & Dengue & Tuberculosis & & \\
\hline & Tuberculosis & Malaria & 0.013 & Sig \\
\hline & & $\begin{array}{c}\text { Liver } \\
\text { Disease }\end{array}$ & 0.016 & Sig \\
\hline \multirow{3}{*}{ MPV } & Dengue & Tuberculosis & 0.006 & $\mathrm{HS}$ \\
\hline & Tuberculosis & Malaria & 0.013 & Sig \\
\hline & & $\begin{array}{c}\text { Liver } \\
\text { Disease }\end{array}$ & 0.433 & \\
\hline \multirow{3}{*}{$\begin{array}{l}\text { P- } \\
\text { LCR }\end{array}$} & Dengue & Tuberculosis & 0.004 & $\mathrm{HS}$ \\
\hline & Tuberculosis & Malaria & 0.010 & Sig \\
\hline & & $\begin{array}{c}\text { Liver } \\
\text { Disease }\end{array}$ & 0.013 & Sig \\
\hline
\end{tabular}

HS- Highly significant, Sig- Significant. 
PDW, MPV, P-LCR were highly significant on comparing values between dengue and tuberculosis. On comparing these values of tuberculosis with malaria and chronic liver disease, p-value was significant.

\section{DISCUSSION}

Platelets play an important role in inflammatory responses; enhance disease severity and mortality in bacterial infections. Platelet activation changes the morphology of platelets including platelet indices. In this study we observed that thrombocytosis patients had low PDW, whereas PDW was higher or normal in patients with thrombocytopenia. Increased MPV and PDW were considered as predictors of poor outcomes among number of infections.[2] MPV is the measure of platelet volume. When platelets have been excessively consumed, bone marrow will produce large amounts of immature platelets, which have larger volume than mature ones. At that time, both newly produced platelets with large volume and mature platelets with small volume are simultaneously present in the blood. Therefore, both MPV and PDW will be increased correspondingly. Platelet indices will provide us with a more comprehensive insight into potential aetiology instead of platelet count alone.[1] A simultaneous reduction of platelet count and PCT indicates that platelets have been excessively consumed. ${ }^{[3]}$

The most common causes of thrombocytopenia in the present study were dengue, malaria, tuberculosis and chronic liver disease. Platelet parameters were analysed in these infections.

Dengue a most important viral infection can present as a self-limiting illness or as severe haemorrhagic shock. Low PLT, MPV and PDW may be used as probable indicators for dengue in endemic area. ${ }^{[4]}$ Dengue patients had a higher PLCR in the present study, which was in concordance with study done by Bashir et al.[4] In the present study, MPV and PDW were normal.

Malaria has been the most common parasitic endemic infection with fatal complications. Early detection by assessing the platelet parameters maybe useful for diagnosis. In this study, MPV was normal in malaria. MPV was higher in patients infected with P. falciparum in Saudi Arabia. [5] In the present study, PCT was not significantly altered in malaria. According to Orathai et al, a combination of platelet count and PCT was the best risk marker for first diagnosis of malaria infection with $99.0 \%$ sensitivity and $95 \%$ specificity. ${ }^{6]}$

In this study tuberculosis patients had a lower MPV, PDW and P-LCR. This was in concordance with study done by Baynes et al, who also found MPV to be low in patients with active tuberculosis.[7] However, Tozkoparan et al found that the MPV was higher in patients with active TB than in non-TB subjects.[7] Sahin et al reported that there was no statistical difference in MPV values between TB patients group and nonTB subjects.[7]

Chronic liver disease caused by Hepatitis virus should be assessed periodically for severity of liver inflammation and fibrosis. According to Ye Pan et al, platelet count and PDW was negatively related to the stage of fibrosis.[8] The present study showed normal platelet indices. MPV is a reflection of pro-inflammatory and prothrombotic events. MPV is reduced where peripheral platelet degradation is increased and where platelet production is impaired. Viral hepatitis and alcoholic liver disease were found to be most common aetiology of cirrhosis patients. The mean MPV values of cirrhotic patients in the study done by Erdem et al were higher than the control group.[9]

In our study on comparison of platelet indices, i.e. PDW and P-LCR across various infections, highly significant $p$ value of 0.004 was observed between dengue and tuberculosis. MPV values between dengue and tuberculosis was statistically significant with a $\mathrm{p}$ value of 0.006 . A statistically significant $\mathrm{p}$ value was also obtained on comparing tuberculosis with Malaria and Chronic liver disease. These pvalues varied with platelet indices as well as with several infections.

\section{CONCLUSION}

Platelet indices have been valuable indicators of illness severity and effective predictors of clinical outcomes.[1] Platelet indices could be easily measured from routine blood examination, that is inexpensive and repeatable. This provides a convenient stratification tool in infections. In this study, high P-LCR was found in malarial and dengue infection. However, tuberculosis had lower platelet indices like MPV, PLCR and PDW. No significant changes were appreciated in chronic liver disease. Platelet indices were known to alter with changes in platelet counts and infections, especially malaria or dengue. Thus, platelet indices such as P-LCR, PDW and MPV can be used as indicators to monitor the outcome of the disease.

\section{REFERENCES}

[1] Navya BN, Patil S, Kariappa TM. Role of platelet parameters in dengue positive cases - an observational study. Int J Health Sci Res 2016;6(6):74-8.

[2] Zhang S, Cui YL, Diao MY, et al. Use of Platelet indices for determining illness severity and predicting prognosis in critically ill patients. Chin Med J (Engl) 2015;128(15):2012-8.

[3] Budak YU, Polat M, Huysal K. The use of platelet indices, plateletcrit, mean platelet volume and platelet distribution width in emergency non-traumatic abdominal surgery: a systematic review. Biochem Med (Zagreb) 2016;26(2):178-93.

[4] Bashir AB, Saeed OK, Mohammed BA, et al. Role of platelet indices in patients with dengue infection in Red Sea State, Sudan. IJSR 2015;4(1):1573-6.

[5] Leal-Santos FA, Silva SB, Crepaldi NP, et al. Altered platelet indices as potential markers of severe and complicated malaria caused by Plasmodium vivax: a cross-sectional descriptive study. Malaria Journal 2013;12:462.

[6] Tangvarasittichai O, Srikong M, Tangvarasittichai S. Platelet count and platelet indices used as potential markers for first malaria infection diagnosis. IJPCR 2016;8(10):1454-8.

[7] Lee MY, Kim YJ, Lee HJ, et al. Mean platelet volume in mycobacterium tuberculosis infection. Article ID 7508763, BioMed Research International 2016;2016:1-4. 
[8] Pan Y, Muheremu A, Wu X, et al. Relationship between platelet parameters and hepatic pathology in patients with chronic hepatitis $B$ infection - a retrospective cohort study of 677 patients. J Int Med Res 2016;44(4):779-86.
[9] Erdem MG, Cil EO, Tukek T, et al. Evaluation of platelet and mean platelet volume levels in patients with liver cirrhosis. Arch Clin Exp Med 2018;3(1):18-21. 